\title{
Cytoreductive Nephrectomy in Metastatic Papillary Renal Cell Carcinoma: Results from the International Metastatic Renal Cell Carcinoma Database Consortium
}

\author{
Jeffrey Graham ${ }^{a}$, J. Connor Wells ${ }^{a}$, Frede Donskov ${ }^{b}$, Jae Lyun Lee ${ }^{c}$, Anna Fraccon ${ }^{d}$, \\ Felice Pasini ${ }^{e}$, Camillo Porta ${ }^{f}$, I. Alex Bowman ${ }^{g}$, Georg A. Bjarnason ${ }^{h}$, D. Scott Ernst ${ }^{i}$, \\ Sun Young Rha ${ }^{j}$, Benoit Beuselinck ${ }^{k}$, Aaron Hansen ${ }^{l}$, Scott A. North ${ }^{m}$, \\ Christian K. Kollmannsberger ${ }^{n}$, Lori A. Wood ${ }^{o}$, Ulka N. Vaishampayan ${ }^{p}$, Sumanta K. Pal ${ }^{q}$, \\ Toni K. Choueiri ${ }^{r, 1}$, Daniel Y.C. Heng ${ }^{a, 1, *}$
}

\footnotetext{
${ }^{\mathrm{a}}$ Tom Baker Cancer Centre, University of Calgary, Calgary, Canada; ${ }^{\mathrm{b}}$ Aarhus University Hospital, Aarhus, Denmark; ' ${ }^{\mathrm{c}}$ University of Ulsan College of Medicine,

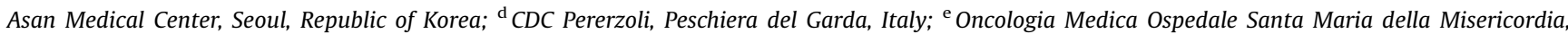
Rovigo, Italy; ${ }^{\mathrm{f}}$ University of Pavia, Pavia, Italy; ${ }^{\mathrm{g}}$ UT Southwestern Medical Center, Dallas, TX, USA; ${ }^{\mathrm{h}}$ Sunnybrook Research Institute, Toronto, Canada; ${ }^{\mathrm{i}}$ London Health Sciences Centre, London, Canada; ${ }^{\mathrm{j}}$ Yonsei University College of Medicine, Seoul, Republic of Korea; ${ }^{\mathrm{k}}$ University Hospitals Leuven, Leuven Cancer Institute, Leuven, Belgium; ${ }^{1}$ Princess Margaret Cancer Centre, Toronto, Canada; ${ }^{\mathrm{m}}$ University of Alberta, Cross Cancer Institute, Edmonton, Canada; ${ }^{\mathrm{n}}$ British Columbia Cancer Agency, Vancouver, Canada; ${ }^{\circ}$ QEII Health Sciences Centre, Halifax, Canada; ${ }^{\mathrm{p}}$ Karmanos Cancer Center, Detroit, MI, USA; ${ }^{\mathrm{Q}}$ City of Hope Comprehensive Cancer Center, Duarte, CA, USA; ${ }^{\mathrm{r}}$ Dana-Farber Cancer Institute, Harvard Medical School, Boston, MA, USA
}

\section{Article info}

Article history:

Accepted March 14, 2019

Associate Editor:

Gianluca Giannarini

Keywords:

Kidney cancer

Metastatic renal cell carcinoma

Papillary

Cytoreductive nephrectomy

\begin{abstract}
Background: There is evidence that cytoreductive nephrectomy ( $\mathrm{CN}$ ) may be beneficial in metastatic renal cell carcinoma (mRCC). This has been studied predominantly in clear-cell RCC, with more limited data on the role of $\mathrm{CN}$ in patients with papillary histology.

Objective: To determine the benefit of $\mathrm{CN}$ in synchronous metastatic papillary RCC. Design, setting, and participants: Using the International Metastatic Renal Cell Carcinoma Database Consortium (IMDC) database, a retrospective analysis was performed for patients with papillary mRCC treated with or without CN.

Outcome measurements and statistical analysis: Median overall survival (OS) and progression-free survival (PFS) were determined for both patient groups. Cox regression analysis was performed to control for imbalances in individual IMDC risk factors.

Results and limitations: In total, 647 patients with papillary mRCC were identified, of whom 353 had synchronous metastatic disease. Of these, 109 patients were treated with $\mathrm{CN}$ and 244 were not. The median follow-up was 57.1 mo (95\% confidence interval [CI $32.9-77.8$ ) and the OS from the start of first-line targeted therapy for the entire cohort was $13.2 \mathrm{mo}$ (95\% CI 12.0-16.1). Median OS for patients with $\mathrm{CN}$ was $16.3 \mathrm{mo}$, compared to $8.6 \mathrm{mo}(p<0.0001)$ in the no-CN group. When adjusted for individual IMDC risk factors, the hazard ratio (HR) of

1 These authors contributed equally to this work.

* Corresponding author. Department of Oncology, Tom Baker Cancer Centre, University of Calgary, 1331 29th Street NW, Calgary T2N 4N2, Canada. Tel.: +1 4036717750.

E-mail address: daniel.heng@ahs.ca (Daniel Y.C. Heng).
\end{abstract}


death for $\mathrm{CN}$ was $0.62(95 \% \mathrm{CI} 0.45-0.85 ; p=0.0031)$. Limitations include the retrospective nature of the analysis.

Conclusions: The use of $\mathrm{CN}$ in patients with $\mathrm{mRCC}$ and papillary histology appears to be associated with better survival compared to no $\mathrm{CN}$ after adjustment for risk criteria. Selection of appropriate candidates for $\mathrm{CN}$ is crucial. A clinical trial in this rare population may not be possible.

Patient summary: In a population of patients with advanced papillary kidney cancer, we found that surgical removal of the primary kidney tumor was associated with better overall survival.

(c) 2019 European Association of Urology. Published by Elsevier B.V. All rights reserved.

\section{Introduction}

Despite impressive advances in treatment, renal cell carcinoma (RCC) remains a significant global health issue, with approximately $30 \%$ of patients presenting with advanced disease [1]. The treatment landscape for metastatic RCC ( $\mathrm{mRCC}$ ) has undergone a dramatic transformation in recent years because of the introduction of molecularly targeted therapies and novel immuno-oncology (IO) agents [2]. Despite these therapeutic advances, the management of mRCC still requires a multimodal approach, with the incorporation of systemic targeted and IO therapies, as well as selected use of radiation therapy and surgical interventions.

The evidence for surgical resection of the primary renal tumor in synchronous metastatic disease is derived from both observational studies and randomized trials. Evidence supporting the use of cytoreductive nephrectomy $(\mathrm{CN})$ comes from data from prospective trials in the era of IFN therapy, as well as retrospective studies performed in the more modern, targeted therapy era. A pooled analysis of two prospective randomized clinical trials demonstrated that $\mathrm{CN}$ followed by IFN treatment was associated with a 5.8-mo increase in overall survival (OS) when compared to IFN alone (13.6 vs $7.8 \mathrm{mo}$ ) [3]. Similarly, retrospective analyses of real-world mRCC data sets have also suggested a survival benefit from $\mathrm{CN}$ among patients treated with targeted therapy $[4,5]$. By contrast, the recently reported phase 3 CARMENA clinical trial demonstrated that targeted therapy with sunitinib alone was noninferior to nephrectomy followed by sunitinib in International mRCC Database Consortium (IMDC) intermediate- and poor-risk patients with clear cell histology [6]. In general, the potential benefits of $\mathrm{CN}$ must be balanced carefully with the possible complications and morbidity of this procedure.

The majority of these studies have focused primarily on clear cell RCC (ccRCC), the most common RCC histological subtype. There are more limited data regarding the role of $\mathrm{CN}$ in non-clear-cell RCC (nccRCC). Within nccRCC pathology, the most common subtype is papillary RCC, accounting for approximately $10-15 \%$ of RCC cases. Papillary RCC has unique biological and clinical features compared to traditional ccRCC histology [7]. This includes more frequent genetic mutations in MET and FH, rather than the VHL alterations seen in ccRCC. Clinically, papillary RCC is characterized by diverse outcomes, with both indolent and aggressive tumors. Thus, the role of $\mathrm{CN}$ in papillary RCC may differ when compared to its ccRCC counterpart. Given this context, this study was designed to determine the benefit of $\mathrm{CN}$ in synchronous metastatic papillary RCC using the IMDC database.

\section{Patients and methods}

\subsection{Patient population}

Patient data were collected retrospectively from 38 international cancer centers included in the IMDC. The IMDC is a large, multi-institutional set comprising data for patients with metastatic RCC [8]. Inclusion criteria for this study were patients with metastatic RCC and papillary histology. We further divided this cohort into patients treated with targeted therapy without a history of nephrectomy, and those treated with $\mathrm{CN}$, defined as nephrectomy performed after diagnosis of synchronous metastatic disease or within $90 \mathrm{~d}$ before diagnosis of metastatic disease [5].

Data were collected using uniform database software and templates. Baseline demographic, clinical, and laboratory data were collected, including variables found to have prognostic significance. Laboratory values were standardized against their respective institution upper limit of normal (ULN) and lower limit of normal (LLN) values as necessary. Outcomes measured included (OS), progression free survival (PFS), and objective response rate (ORR) to first-line therapy. The data included patients accrued between 2005 and October 2017. This study received institutional review board approval from each participating center.

\subsection{Statistical analysis}

The primary outcome of this study was OS, defined as time from initiation of first-line targeted therapy to the date of death or censored at last follow-up. A secondary outcome was PFS, defined as time from initiation of targeted therapy to the date of progression, drug cessation, or censored at last follow-up. Median OS and PFS distributions were estimated using the Kaplan-Meier method. Cox proportional hazards regression was used to determine hazard ratios (HRs) after adjustment for baseline prognostic variables. This included adjusting for the IMDC prognostic factors: hemoglobin below the LLN, corrected calcium greater than the ULN, neutrophils above the ULN, platelets greater than the ULN, Karnofsky performance status (KPS) $<80 \%$, and time from diagnosis to treatment of $<1 \mathrm{yr}$. The IMDC prognostic factors have been validated in metastatic papillary RCC [9]. Adjusted HRs and corresponding $p$ values are reported. Statistical analyses were performed with SAS v.9.4 (SAS Institute, Cary, NC, USA), with statistical significance set at $p<0.05$ (twosided test). 


\section{Results}

At the time of analysis, a total of 8798 patients with mRCC were included in the overall IMDC data set. Within this population, 647 patients were identified as having papillary histology. Of this subset, 538 underwent nephrectomy. Those who had a prior nephrectomy and then subsequently developed metastatic disease were excluded $(n=294)$ to isolate patients with synchronous metastatic disease. The final numbers for the analysis included 109 patients without $\mathrm{CN}$ and 244 with $\mathrm{CN}$. The median number of patients included by the contributing institutions was nine. The median followup for all patients was $57.1 \mathrm{mo}$ (95\% confidence interval [CI] 32.9-77.8). The median OS from the start of first-line targeted therapy for the entire cohort was 13.2 mo (95\% CI 12.0-16.1).

Table 1 compares baseline characteristics for the $\mathrm{CN}$ and no$\mathrm{CN}$ groups. Table 2 summarizes the type of targeted therapy received and the number of subsequent lines of therapy. Patients who underwent $\mathrm{CN}$ were more likely to be younger ( $p=0.0001)$ with better performance status $(p=0.0231)$. There was also a higher number of bone metastases in the $\mathrm{CN}$ group $(p=0.0281)$. The proportions of type 1 and type 2 histology were similar between the two groups $(p=0.902)$. Sunitinib was the most common first-line targeted therapy used in both groups. The ORR to first-line therapy did not differ between $\mathrm{CN}$ and no-CN (12\% vs 5.9\%; $p=0.2847$ ).

The median OS for the $\mathrm{CN}$ group was $16.3 \mathrm{mo}$, compared to $8.6 \mathrm{mo}$ in the no-CN group (Fig. $1 ; p<0.0001$ ). On multivariable analysis adjusted for IMDC prognostic factors, the HR for death was 0.62 (95\% CI 0.45-0.85; $p=0.0031$ ), translating into a $38 \%$ decrease in the risk of death with $\mathrm{CN}$. After adjusting for additional prognostic imbalances including the IMDC criteria, age, and the presence of bone metastases, the HR improved to 0.55 (95\% CI 0.39-0.78; $p=0.0006)$. With regard to secondary outcomes, PFS also
Table 2 - Baseline treatment characteristics for the $\mathrm{CN}$ and no-CN groups.

\begin{tabular}{lrrr}
\hline & $\begin{array}{c}\text { No CN } \\
(N=109)\end{array}$ & $\begin{array}{c}\text { CN } \\
(N=244)\end{array}$ & $p$ value \\
\hline First-line therapy, $n / N(\%)$ & & & \\
$\quad$ Sunitinib & $59 / 109(54)$ & $134 / 244(55)$ & \\
Sorafenib & $3 / 109(2.8)$ & $31 / 244(13)$ & \\
Temsirolimus & $25 / 109(23)$ & $31 / 244(13)$ & \\
Pazopanib & $12 / 109(11)$ & $19 / 244(7.8)$ & \\
Other & $10 / 109(9.1)$ & $29 / 244(12)$ & \\
Second-line therapy, $n / N(\%)$ & $43 / 109(39)$ & $137 / 244(56)$ & 0.0037 \\
Third-line therapy, $n / N(\%)$ & $17 / 109(16)$ & $58 / 244(24)$ & 0.0828 \\
\hline Fourth-line therapy, $n / N(\%)$ & $4 / 109(3.7)$ & $16 / 244(6.6)$ & 0.2783 \\
\hline CN = cytoreductive nephrectomy. & & \\
\hline
\end{tabular}

appeared to be prolonged in the $\mathrm{CN}$ group at $5.1 \mathrm{mo}$, compared to $3.4 \mathrm{mo}$ in the no-CN group (Fig. $2 ; p=0.0344$ ). After adjusting for the IMDC factors, the HR for PFS was 0.82 (95\% CI 0.61-1.10; $p=0.1822$ ). When age and the presence of bone metastases were added to the regression model, the HR for PFS was 0.73 (95\% CI 0.53-1.01; $p=0.0555)$. For comparative purposes, we performed an analysis of $\mathrm{CN}$ versus no-CN in the ccRCC population. In this ccRCC subset, median OS was $21.8 \mathrm{mo}$ in the $\mathrm{CN}$ group, compared to 10.0 mo in the no-CN group ( $p<0.0001)$.

We also performed a subgroup analysis by dividing the $\mathrm{CN}$ cohort into immediate systemic therapy (defined as within $90 \mathrm{~d}$ of surgery) and delayed systemic therapy (defined as $>90 \mathrm{~d}$ from $\mathrm{CN}$ ) groups. There was no significant survival difference between these two subgroups.

\section{Discussion}

The role of $\mathrm{CN}$ in the contemporary management of advanced kidney cancer has been well studied in retrospective studies,

Table 1 - Baseline characteristics for the $\mathrm{CN}$ and non- $\mathrm{CN}$ groups.

\begin{tabular}{|c|c|c|c|}
\hline & $\begin{array}{c}\text { No } C N \\
(N=109)\end{array}$ & $\begin{array}{c}C N \\
(N=244)\end{array}$ & $p$ value \\
\hline Male, $n(\%)$ & $86 / 109$ (79) & $173 / 244(71)$ & 0.1163 \\
\hline Median age (yr) & 67 & 59 & 0.0001 \\
\hline Karnofsky performance status $<80 \%, n / N(\%)$ & $28 / 90(31)$ & $41 / 214(19)$ & 0.0231 \\
\hline Diagnosis to targeted therapy $<1 \mathrm{yr}, n / N(\%)$ & $99 / 109(91)$ & $204 / 244(84)$ & 0.0723 \\
\hline Calcium > ULN, $n / N(\%)$ & $16 / 89(18)$ & $30 / 193(16)$ & 0.6072 \\
\hline Hemoglobin <LLN, $n / N(\%)$ & $69 / 99(70)$ & $136 / 216(63)$ & 0.2445 \\
\hline Neutrophils >ULN, $n / N(\%)$ & $25 / 98(26)$ & $38 / 209(18)$ & 0.1383 \\
\hline Platelets $>$ ULN, $n / N(\%)$ & $26 / 98(27)$ & $42 / 213(20)$ & 0.1769 \\
\hline Histologic subtype, $n / N(\%)$ & & & 0.902 \\
\hline Type 1 & $6 / 41(14.6)$ & $13 / 94(13.8)$ & \\
\hline Type 2 & $35 / 41(85.4)$ & $81 / 94(86.2)$ & \\
\hline Liver metastases, $n / N(\%)$ & $21 / 105(20)$ & $50 / 207(24 \%)$ & 0.4082 \\
\hline Bone metastases, $n / N(\%)$ & $30 / 107(28)$ & $86 / 212(41 \%)$ & 0.0281 \\
\hline Brain metastases, $n / N(\%)$ & $3 / 106(2.8)$ & $5 / 204(2.5 \%)$ & 0.8417 \\
\hline More than one site of metastasis, $n / N(\%)$ & $87 / 108(81)$ & $169 / 222(76.1 \%)$ & 0.3653 \\
\hline IMDC risk group, $n / N(\%)$ & & & 0.2627 \\
\hline Favorable risk & $4 / 78(5.1)$ & $10 / 175(5.7)$ & \\
\hline Intermediate risk & $37 / 78$ (47) & $101 / 175(58)$ & \\
\hline Poor risk & $37 / 78(47)$ & $64 / 175(37)$ & \\
\hline
\end{tabular}




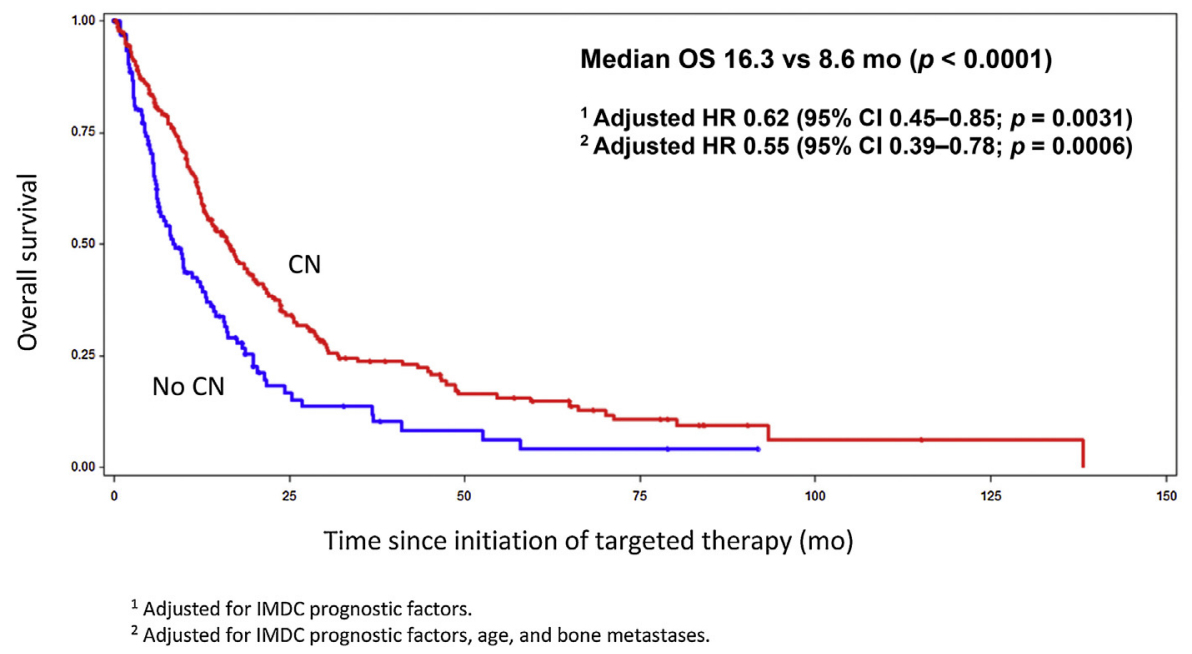

Fig. 1 - Kaplan-Meier curve for overall survival (OS) for the $\mathrm{CN}$ and no- $\mathrm{CN}$ groups. $\mathrm{CN}=$ cytoreductive nephrectomy; $\mathrm{HR}=$ hazard ratio; $\mathrm{CI}=$ confidence interval; IMDC = International Metastatic Renal Cell Carcinoma Database Consortium.

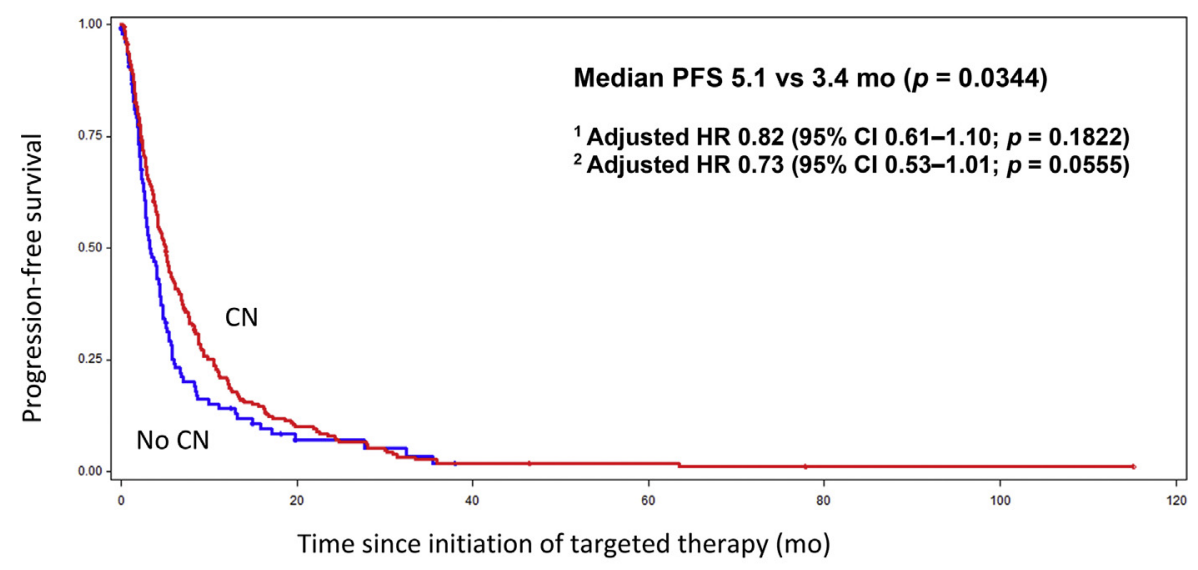

${ }^{1}$ Adjusted for IMDC prognostic factors.

${ }^{2}$ Adjusted for IMDC prognostic factors, age, and bone metastases.

Fig. 2 - Kaplan-Meier curve for progression-free survival (PFS) for the $\mathrm{CN}$ and no-CN groups. $\mathrm{CN}=$ cytoreductive nephrectomy; HR = hazard ratio; $\mathrm{CI}=$ confidence interval; IMDC = International Metastatic Renal Cell Carcinoma Database Consortium.

analyses of real-world data, and randomized controlled trials. Prior retrospective analyses of different data sets, including the IMDC and the National Cancer Data Base, have consistently revealed that $\mathrm{CN}$ appears to offer a survival benefit in ccRCC $[5,10]$.

Although more limited than the literature on ccRCC, previous retrospective analyses have also suggested a benefit of $\mathrm{CN}$ in nccRCC [11,12]. It is important to note that nccRCC represents a diverse spectrum of pathological and clinical entities, with papillary RCC being the most common subtype. Aizer et al. [12] analyzed the role of $\mathrm{CN}$ in metastatic nccRCC using the Surveillance, Epidemiology, and End Results (SEER) database. In this more heterogeneous population, $\mathrm{CN}$ was associated with lower cancerspecific and all-cause mortality (HR 0.45, 95\% CI 0.37-0.55; $p<0.001$ ). Marchioni et al. [11] also examined the role of $\mathrm{CN}$ in nccRCC patients using an updated analysis of the SEER database. They again demonstrated a cancer-specific mortality benefit with CN (HR 0.38, 95\% CI 0.30-0.47; $p<0.001)$. In a subgroup analysis looking at patients with papillary RCC, CN was also associated with better cancerspecific mortality (HR 0.40, 95\% CI 0.30-0.53; $p<0.001$ ). None of these studies adjusted for potential confounders such as IMDC criteria, specifically for the time between diagnosis and systemic therapy.

The results of our analysis for patients with exclusively papillary RCC are consistent with these findings. In contrast to the SEER database, the IMDC is able to control for more specific prognostic variables, including performance status at initiation of systemic therapy and biochemical parameters. In our study, the group receiving $\mathrm{CN}$ were younger and had better performance status, probably reflecting an element of bias in surgical selection. We demonstrated that patients undergoing $\mathrm{CN}$ had significantly better $\mathrm{OS}$, 
even when controlling for prognostic imbalances between these groups, including performance status, age, and the IMDC risk factors. A PFS benefit in favor of $\mathrm{CN}$ did not meet statistical significance in the multivariable analysis. The proportion of type 1 and type 2 histology was similar between these two groups, which is important given the clinical significance of these subtypes. To the best of our knowledge, our study represents the largest multivariable analysis exploring $\mathrm{CN}$ in papillary RCC. Given that advanced papillary RCC typically has lower response rates to traditional VEGF targeted therapies, it may be even more important to achieve local tumor control and cytoreduction in this tumor subtype [13].

In contrast to these findings, the recently reported CARMENA randomized controlled trial demonstrated that sunitinib therapy alone appeared to be noninferior to $\mathrm{CN}$ followed by sunitinib in patients with intermediate and poor risk [6]. The results in the sunitinib-alone group were noninferior to those in the $\mathrm{CN}$ followed by sunitinib group with regard to OS (stratified HR for death, $0.89,95 \% \mathrm{Cl} 0.71-$ 1.10 ; upper bound of the $95 \% \mathrm{CI}$ for noninferiority, $\leq 1.20$ ).

Importantly, the CARMENA trial excluded patients with nccRCC and by definition excluded patients with favorable risk. In addition, patients randomized in this trial may not accurately reflect ideal $\mathrm{CN}$ candidates in a real-world setting, possibly because of a perceived lack of clinical equipoise [14]. This was evident in the slow accrual to the trial, which required a reduction in its sample size, and the unusually high number of IMDC poor-risk patients in the cohort (43\%). Prior studies using the IMDC database have suggested that patients with poorer risk, including those with four or more IMDC risk factors, do not appear to derive benefit from $\mathrm{CN}$ [5]. Thus, utilization of $\mathrm{CN}$ in this poor-risk population may have helped the trial meet its noninferiority endpoint. The efficacy of systemic therapy in papillary RCC is suboptimal, highlighting the importance of $\mathrm{CN}$ as a potential therapeutic strategy in metastatic disease.

In general, $\mathrm{CN}$ may be considered for patients with good performance status and limited or slow-growing metastatic disease. Symptomatic patients with ongoing hematuria or flank pain may also benefit from $\mathrm{CN}$. It is important to discuss these cases in a multidisciplinary tumor board to consider $\mathrm{CN}$ feasibility, comorbidities, and an estimate of the overall prognosis to gain an insight into whether the patients will benefit from surgery. Thus, for patients with papillary RCC, careful patient selection for $\mathrm{CN}$ is crucial.

Another randomized trial (SURTIME trial) randomized mRCC patients to immediate $\mathrm{CN}$ followed by sunitinib versus delayed $\mathrm{CN}$ after three cycles of initial sunitinib $[15,16]$. A trend towards better OS for those with deferred nephrectomy was observed. This may in part be due to patient selection after initial sunitinib, in that patients who had a favorable tumor response went on to $\mathrm{CN}$, while $\mathrm{CN}$ was aborted for those who had progressive disease. This trial may give treating physicians the comfort that delaying a decision on $\mathrm{CN}$ may be reasonable, especially for patients with intermediate or poor risk. Similar to the CARMENA trial, SURTIME included patients with ccRCC only, and thus may not be fully generalizable to those with nccRCC or papillary histology.

Limitations of our study include the retrospective nature of the analysis and the presence of inherent, unmeasured confounders that could not be adjusted for, despite multivariable analysis. Selection bias is another important limitation that is intrinsic to the retrospective design of the study. The IMDC uses a consecutive patient series to minimize selection bias. Given these limitations, a causal relationship between $\mathrm{CN}$ and survival cannot be definitively established. There was no central pathology review, as this was a multi-institutional study. Our data set only includes patients who are receiving active systemic therapy, and thus does not include patients with mRCC undergoing active surveillance. Owing to low patient numbers, we were unable to perform a subgroup analysis looking at the relationship between a number of individual IMDC factors and benefit from $\mathrm{CN}$. We were also unable to account for outcomes such as perioperative mortality and surgeryrelated morbidity.

\section{Conclusions}

In summary, in our data set it appears that $\mathrm{CN}$ is associated with better survival in metastatic papillary RCC, even when controlling for known prognostic imbalances between the groups. Given the lower response rates to targeted therapy in papillary RCC, achieving local tumor control may be particularly important in this pathological subtype. As in the more commonly studied ccRCC population, selection of patients for $\mathrm{CN}$ will be critical. Unfortunately, a prospective clinical trial in this rare subgroup may not be possible. As the treatment landscape for mRCC evolves, it will be important to reevaluate the role of $\mathrm{CN}$ in papillary RCC, particularly in combination with novel IO and MET targeted therapy.

Author contributions: Daniel Y.C. Heng had full access to all the data in the study and takes responsibility for the integrity of the data and the accuracy of the data analysis.

Study concept and design: All authors.

Acquisition of data: All authors.

Analysis and interpretation of data: Heng, Graham.

Drafting of the manuscript: Heng, Graham.

Critical revision of the manuscript for important intellectual content: All authors.

Statistical analysis: Heng, Graham.

Obtaining funding: None.

Administrative, technical, or material support: Heng, Graham. Supervision: Heng.

Other: None.

Financial disclosures: Daniel Y.C. Heng certifies that all conflicts of interest, including specific financial interests and relationships and affiliations relevant to the subject matter or materials discussed in the manuscript (eg, employment/affiliation, grants or funding, consultancies, honoraria, stock ownership or options, expert testimony, royalties, or patents filed, received, or pending), are the following: Jae Lyun Lee has received honoraria from Astellas Pharma, Bristol-Myers Squibb, Novartis, and Pfizer; has acted in a consulting or advisory role for Astellas Pharma, AstraZeneca, and Eisai; and has received research funding from BristolMyers Squibb, Exelixis, Janssen, Novartis, Pfizer, and Roche/Genentech. 
Camillo Porta has acted in a consulting or advisory role for Bristol-Myers Squibb, Eisai, EUSA Pharma, Ipsen, Janssen, Novartis, Peloton Therapeutics, and Pfizer. Georg A. Bjarnason has received honoraria from and acted in a consulting or advisory role for Bristol-Myers Squibb, Novartis, and Pfizer; has received institutional research funding from Merck and Pfizer; and has received travel and accommodation expenses from Novartis and Pfizer. D. Scott Ernst has acted in a consulting or advisory role for AstraZeneca/MedImmune, Bristol-Myers Squibb, Merck, Novartis Canada Pharmaceuticals, and Roche Canada. Benoit Beuselinck has received honoraria form Amgen, Bayer, Janssen, and Pfizer. Scott A. North has received honoraria from Astellas Pharma, Janssen-Ortho, Novartis, Pfizer, and Sanofi Canada; has acted in a consulting or advisory role for Astellas Pharma, AstraZeneca, Janssen Oncology, Merck, Novartis, Pfizer, Roche Canada, and Sanofi Canada; and has received institutional research funding from Astellas Pharma, AstraZeneca, Janssen, Novartis Canada Pharmaceuticals, Roche Canada, and Sanofi Canada. Christian K. Kollmannsberger has received honoraria from Bristol-Myers Squibb, Novartis, and Pfizer; has acted in a consulting or advisory role for Astellas Pharma, Bristol-Myers Squibb, Novartis, Pfizer, and Seattle Genetics; and has received travel and accommodation expenses from Novartis and Pfizer. Lori A. Wood has received institutional research funding from Aragon Pharmaceuticals, AstraZeneca, Bristol-Myers Squibb, Exelixis, Merck, Novartis, Pfizer, and Roche Canada. Ulka N. Vaishampayan has received honoraria from Astellas Pharma, Bayer, Bristol-Myers Squibb, Exelixis, Genentech, Janssen, Novartis, Pfizer, and Sanofi; has acted in a consulting or advisory role and participated in a speakers' bureau for Astellas Pharma, Bayer, Bristol-Myers Squibb, Exelixis, Genentech/Roche, and Pfizer; and has received research funding from Astellas Pharma, Bristol-Myers Squibb, Exelixis, Novartis, and Pfizer. Sumanta K. Pal has received honoraria from Astellas Pharma, Medivation, and Novartis; has acted in a consulting or advisory role for Astellas Pharma, Aveo, BristolMyers Squibb, Eisai, Exelixis, Genentech, Ipsen, Myriad Pharmaceuticals, Novartis, and Pfizer; and has received research funding from Medivation. Toni K. Choueiri has received honoraria from NCCN and UpToDate; has acted in a consulting or advisory role for Alligent, AstraZeneca, Bayer, Bristol-Myers Squibb, Cerulean Pharma, Eisai, Exelixis, Foundation Medicine, GlaxoSmithKline, Merck, Novartis, Peloton Therapeutics, Pfizer, Prometheus Laboratories, and Roche/Genentech; has received institutional research funding from Agensys, AstraZeneca, Bristol-Myers Squibb, Celldex, Exelixis, GlaxoSmithKline, Merck, Novartis, Peloton Therapeutics, Pfizer, Roche/Genentech, and TRACON Pharma; and has received travel and accommodation expenses when acting in an advisory or consulting role. Daniel Y.C. Heng has acted in a consulting or advisory role for Astellas Pharma, Bristol-Myers Squibb, Janssen, Novartis, and Pfizer; and has received institutional research funding from BristolMyers Squibb, Exelixis, Novartis, and Pfizer. The remaining authors have nothing to disclose.

Funding/Support and role of the sponsor: None.

\section{References}

[1] Chow W-H, Devesa SS. Contemporary renal cell cancer epidemiology. Cancer J 2008;14:288-301.
[2] Choueiri TK, Motzer RJ. Systemic therapy for metastatic renal-cell carcinoma. N Engl J Med 2017;376:354-66.

[3] Flanigan RC, Mickisch G, Sylvester R, Tangen C, Van Poppel H, Crawford ED. Cytoreductive nephrectomy in patients with metastatic renal cancer: a combined analysis. J Urol 2004;171:1071-6.

[4] Choueiri TK, Xie W, Kollmannsberger C, et al. The impact of cytoreductive nephrectomy on survival of patients with metastatic renal cell carcinoma receiving vascular endothelial growth factor targeted therapy. J Urol 2011;185:60-6.

[5] Heng DYC, Wells JC, Rini BI, et al. Cytoreductive nephrectomy in patients with synchronous metastases from renal cell carcinoma: results from the International Metastatic Renal Cell Carcinoma Database Consortium. Eur Urol 2014;66:704-10.

[6] Méjean A, Ravaud A, Thezenas S, et al. Sunitinib alone or after nephrectomy in metastatic renal-cell carcinoma. N Engl J Med 2018;379:417-27. http://dx.doi.org/10.1056/NEJMoa1803675.

[7] Linehan WM, Spellman PT, Ricketts CJ, et al. Comprehensive molecular characterization of papillary renal-cell carcinoma. N Engl J Med 2016;374:135-45.

[8] Heng DYC, Xie W, Regan MM, et al. Prognostic factors for overall survival in patients with metastatic renal cell carcinoma treated with vascular endothelial growth factor-targeted agents: results from a large, multicenter study. J Clin Oncol 2009;27:5794-9.

[9] Wells JC, Donskov F, Fraccon AP, et al. Characterizing the outcomes of metastatic papillary renal cell carcinoma. Cancer Med 2017;6:902-9.

[10] Hanna N, Sun M, Meyer CP, et al. Survival analyses of patients with metastatic renal cancer treated with targeted therapy with or without cytoreductive nephrectomy: a National Cancer Data Base study. J Clin Oncol 2016;34:3267-75.

[11] Marchioni M, Bandini M, Preisser F, et al. Survival after cytoreductive 363 nephrectomy in metastatic non-clear cell renal cell carcinomapatients: 364 a population-based study. Eur Urol Focus 2017. http://dx.doi.org/10.1016/j.euf.2017.11.012.

[12] Aizer AA, Urun Y, McKay RR, et al. Cytoreductive nephrectomy in patients with metastatic non-clear-cell renal cell carcinoma (RCC). BJU Int 2014;113:E67-74.

[13] Ravaud A, Oudard S, De Fromont M, et al. First-line treatment with sunitinib for type 1 and type 2 locally advanced or metastatic papillary renal cell carcinoma: a phase II study (SUPAP) by the French Genitourinary Group (GETUG). Ann Oncol 2015;26:1123-8.

[14] Motzer RJ, Russo P. Cytoreductive nephrectomy-patient selection is key. N Engl J Med 2018;379:481-2. http://dx.doi.org/10.1056/ NEJMe1806331.

[15] Bex A, Mulders P, Jewett M, et al. Immediate versus deferred cytoreductive nephrectomy $(\mathrm{CN})$ in patients with synchronous metastatic renal cell carcinoma (mRCC) receiving sunitinib (EORTC 30073 SURTIME). Ann Oncol 2017;28(Suppl. 5):mdx440.030.

[16] Bex A, Mulders P, Jewett M, et al. Comparison of immediate vs deferred cytoreductive nephrectomy in patients with synchronous metastatic renal cell carcinoma receiving sunitinib: the SURTIME randomized clinical trial. JAMA Oncol 2019;5:164-70. 